\title{
Sampled-Data Control of Spacecraft Rendezvous with Discontinuous Lyapunov Approach
}

\author{
Zhuoshi Li, ${ }^{1}$ Ming Liu, ${ }^{1}$ Hamid Reza Karimi, ${ }^{2}$ and Xibin Cao ${ }^{1}$ \\ ${ }^{1}$ Research Center of Satellite Technology, Harbin Institute of Technology, Harbin 150001, China \\ ${ }^{2}$ Department of Engineering, Faculty of Technology and Science, University of Agder, 4898 Grimstad, Norway \\ Correspondence should be addressed to Ming Liu; robustcontrol23@gmail.com
}

Received 23 May 2013; Accepted 19 August 2013

Academic Editor: Rongni Yang

Copyright (c) 2013 Zhuoshi Li et al. This is an open access article distributed under the Creative Commons Attribution License, which permits unrestricted use, distribution, and reproduction in any medium, provided the original work is properly cited.

\begin{abstract}
This paper investigates the sampled-data stabilization problem of spacecraft relative positional holding with improved Lyapunov function approach. The classical Clohessy-Wiltshire equation is adopted to describe the relative dynamic model. The relative position holding problem is converted into an output tracking control problem using sampling signals. A time-dependent discontinuous Lyapunov functionals approach is developed, which will lead to essentially less conservative results for the stability analysis and controller design of the corresponding closed-loop system. Sufficient conditions for the exponential stability analysis and the existence of the proposed controller are provided, respectively. Finally, a simulation result is established to illustrate the effectiveness of the proposed control scheme.
\end{abstract}

\section{Introduction}

In the research area of advanced astronautic missions, spacecraft autonomous rendezvous and docking has been one of the most significant problems over the past few decades. Generally speaking, before completing the final docking action between the chaser spacecraft and the target spacecraft, the chaser spacecraft is forbidden to maintain at a fixed relative position around the target and prepares to implement the final docking action and/or other special missions [1-3]. This procedure is the so-called "relative position holding." In recent years, a few results have been developed in the existing literatures to investigate the problems of autonomous relative motion for multiple spacecrafts $[4,5]$. Among these existing approaches, one of the most popular mathematical tool is the so-called Clohessy-Wiltshire (C-W) equation. This model is introduced by Clohessy and Wiltshire in 1960 [6] and has been broadly applied to address the problem of relative motion between two neighboring spacecrafts $[7,8]$.

On the other hand, in modern industrial process, one or more computers are always employed as digital controllers to control continuous-time plant [9-12]. Under this setting, digital computers are required to sample and quantize a continuous-time analog signal to evaluate a discrete-time sampled-data signal [13-15], based on which a discretetime control input signal is designed [16-19]. This discretetime signal is further converted into a continuous-time signal based on a zero-order hold equipment in the actuator side [20-24]. Subsequently, the corresponding sampleddata systems contain both continuous-time and discretetime signals in the continuous-time domain [25-28]. Due to its significance, over the past decade the problems of stabilization and filtering of sampled-data systems have been investigated extensively; see for instance, $[29,30]$ and the reference therein.

It is worth pointing out that there exist three main methodologies for the stabilization and filtering problems of sampled-data systems [31,32], which are lifting technique method, impulsive model method, and input delay method, respectively. Among these existing approaches, input delay approach is the latest established approach, wherein the sampled-data system is modeled as a continuous-time system with time-varying delay existing in the control input. A great number of results based on this approach have been proposed in the existing literatures [33, 34]. Among these existing results, the time-independent Lyapunov function approach 
presented by Fridman et al. [34] has been proved to be effective to deal with the input delay. Based on the timeindependent Lyapunov approach, an improved Lyapunov approach, namely, time-dependent Lyapunov approach has been also developed by Fridman [33] and Naghshtabrizi et al. [35]. Compared with the time-independent Lyapunov approach, the structure characteristic of the time-dependent Lyapunov approach can guarantee that its value does not increase in each sampling time despite the jumping behavior of the sampled signals and thus can reduce the design conservativeness. This method is later extended to deal with filtering problem of Itô stochastic systems with signal sampling and quantization [36].

It should be pointed out that, in practical sampled-data systems of spacecraft rendezvous, there also exists jumping behavior of the sampled signal at each sampling time instant, and the values of the sampled signal increase abruptly at these time instants. Therefore, it is desirable to reduce the conservativeness of the controller design of sampled-data spacecraft rendezvous systems by developing new control schemes. Motivated by the aforementioned time-dependent Lyapunov approach, in this paper, we consider the sampleddata control problem for relative position holding of spacecraft rendezvous. In this study, signal sampling, control input limitation, and $H_{\infty}$ performance are considered together in a unified framework. The discontinuous Lyapunov functions approach is adopted for the controller design; also we introduce free-weight matrices in the Lyapunov functions, and it will be shown that the obtained results are less conservative then those in the existing work.

The remainder of this paper is organized as follows. In Section 2, the problem of sampled-data control of relative position holding of spacecraft rendezvous is formulated. In Section 3, the sufficient condition for the exponential stability of the sampled-data closed-loop system is provided, based on which the sufficient for the existence of the sampled-data controller is given. Finally, a numerical example is presented in Section 4 to demonstrate the feasibility of the proposed method.

\section{Problem Formulation}

In the orbital coordinate frame of chaser and target spacecrafts, it is well defined that the origin attaches to the mass center of the target spacecraft, $x$-axis is along the vector from the Earth center to the origin, $y$-axis is along the target orbit circumference, $z$-axis completes the right-handed frame, $r_{1}$ denotes the radius of the target circular orbit, $\mu$ denotes the gravitational parameter of the earth, $w$ denotes the angle velocity of the target, and $w=\left(\mu / r_{1}^{3}\right)^{1 / 2}$.

Based on the Newton motion theory, the relative dynamic model of chaser and target spacecrafts can be modeled by the following $\mathrm{C}-\mathrm{W}$ equations:

$$
\begin{aligned}
\ddot{x}_{c}(t)= & 2 w \dot{y}_{c}(t)+w^{2}\left(r_{1}+x_{c}(t)\right) \\
& -\frac{\mu\left(r_{1}+x_{c}(t)\right)}{\left[\left(r_{1}+x_{c}(t)\right)^{2}+y_{c}^{2}(t)+z_{c}^{2}(t)\right]^{3 / 2}}+a_{x},
\end{aligned}
$$

$$
\begin{aligned}
& \ddot{y}_{c}(t)=-2 w \dot{x}_{c}(t)+w^{2} y_{c}(t) \\
&-\frac{\mu y_{c}(t)}{\left[\left(r_{1}+x_{c}(t)\right)^{2}+y_{c}^{2}(t)+z_{c}^{2}(t)\right]^{3 / 2}}+a_{y}, \\
& \ddot{z}_{c}(t)=-\frac{\mu y_{c}(t)}{\left[\left(r_{1}+x_{c}(t)\right)^{2}+y_{c}^{2}(t)+z_{c}^{2}(t)\right]^{3 / 2}}+a_{z},
\end{aligned}
$$

where $x_{c}(t), y_{c}(t)$, and $z_{c}(t)$ are the components of the relative position in corresponding axes and $a_{i}(i=x, y, z)$ is the $i$ th component of the control input vector. By the taylor expansion and linearization approach, the linearized equation around the null solution for (1) can be given by

$$
\begin{gathered}
\ddot{x}_{c}(t)-2 w \dot{y}_{c}(t)-3 w^{2} x_{c}(t)=\frac{1}{m}\left(T_{x}+d_{x}\right), \\
\ddot{y}_{c}(t)+2 w \dot{x}_{c}(t)=\frac{1}{m}\left(T_{y}+d_{y}\right), \\
\ddot{z}_{c}(t)+w^{2} z_{c}(t)=\frac{1}{m}\left(T_{z}+d_{z}\right) .
\end{gathered}
$$

In system (2), $m$ is the mass of the chaser, $T_{i}(i=x, y, z)$ is the relative motion dynamic, and $d_{i}, i=x, y, z$ is the $i$ th component of the external disturbances. We define the following vector and matrices:

$$
\begin{aligned}
& x(t)=\left[\begin{array}{c}
x_{c}(t) \\
y_{c}(t) \\
z_{c}(t) \\
\dot{x}_{c}(t) \\
\dot{y}_{c}(t) \\
\dot{z}_{c}(t)
\end{array}\right], \quad u(t)=\left[\begin{array}{c}
T_{x}(t) \\
T_{y}(t) \\
T_{z}(t)
\end{array}\right], \\
& d(t)=\left[\begin{array}{l}
d_{x}(t) \\
d_{y}(t) \\
d_{z}(t)
\end{array}\right], \quad y(t)=\left[\begin{array}{l}
x_{c}(t) \\
y_{c}(t) \\
z_{c}(t)
\end{array}\right], \\
& A=\left[\begin{array}{cccccc}
0 & 0 & 0 & 1 & 0 & 0 \\
0 & 0 & 0 & 0 & 1 & 0 \\
0 & 0 & 0 & 0 & 0 & 1 \\
3 w^{2} & 0 & 0 & 0 & 2 w & 0 \\
0 & 0 & 0 & -2 w & 0 & 0 \\
0 & 0 & -w^{2} & 0 & 0 & 0
\end{array}\right]
\end{aligned}
$$




$$
B=\frac{1}{m}\left[\begin{array}{lll}
0 & 0 & 0 \\
0 & 0 & 0 \\
0 & 0 & 0 \\
1 & 0 & 0 \\
0 & 1 & 0 \\
0 & 0 & 1
\end{array}\right]
$$

Then the C-W equation (2) can be described equivalently as follows:

$$
\begin{gathered}
\dot{x}(t)=A x(t)+B u(t)+B d(t), \\
y(t)=C x(t) .
\end{gathered}
$$

As a result, the specific relative motion of the chaser and target can be realized via designing effective control strategy $u(t)$ for system (5).

In this paper, we consider the case that the signals are sampled before transmitted to the controller side. Define $\mathscr{T} \triangleq$ $\left\{t_{1}, t_{2}, t_{3}, \ldots\right\}$ as a strictly increasing sequence of sampling times in $\left(t_{0}, \infty\right)$ for initial time $t_{0}=0$. Suppose that the sampling internals between any two sequent sampling instants are bounded by

$$
t_{k+1}-t_{k} \leq h, \quad k=1,2, \ldots,
$$

where $h>0$ is a known constant. Then, the control vector is assumed to be of the following form at the sampling instant $t_{k}$ with zero-order hold $(\mathrm{ZOH})$ :

$$
u(t)=K x\left(t_{k}\right)
$$

During the orbital transfer process, the thrust constraint can be described as

$$
\left|u_{i}\left(t_{k}\right)\right| \leqslant u_{i, \max } \quad(i=x, y, z),
$$

where $u_{i}\left(t_{k}\right)$ is the control input thrust along the $i$ th axis at the sampling instant $k$ and $u_{i \text {,max }}$ is the maximum allowed thrust along the $i$ th axis.

On the other hand, the desired holding position can be treated as a reference output $y_{r}$ of (5). Therefore, our object is thus to propose a sampled-data control strategy (7), such that the closed-loop system for plant (5) is exponentially stable and the output $y(t)$ tracks the reference signal $y_{r}$ without steady-state error. Subsequently, the problem of relative control holding is converted into an output tracking control problem. To achieve

$$
\lim _{t \rightarrow+\infty} y(t)=y_{r}
$$

we define the output error

$$
e(t)=\int_{0}^{t} y_{e}(t) d t
$$

Also, we define the following augmented system

$$
\dot{\xi}(t)=\bar{A} \xi(t)+\bar{B} u\left(t_{k}\right)+\bar{B}_{1} \bar{d}(t)
$$

with

$$
\begin{gathered}
\xi(t)=\left[\begin{array}{l}
x(t) \\
e(t)
\end{array}\right], \quad \bar{d}(t)=\left[\begin{array}{c}
d(t) \\
y_{r}
\end{array}\right], \\
\bar{A}=\left[\begin{array}{ll}
A & 0_{6 \times 3} \\
C & 0_{3 \times 3}
\end{array}\right], \quad \bar{B}=\left[\begin{array}{c}
B \\
0_{3 \times 3}
\end{array}\right], \\
\bar{B}_{1}=\left[\begin{array}{cc}
B & 0_{6 \times 3} \\
0_{3 \times 3} & -I_{3 \times 3}
\end{array}\right], \\
\bar{C}_{1}=\left[\begin{array}{ll}
C & 0_{3 \times 3}
\end{array}\right], \quad \bar{C}_{2}=\left[\begin{array}{ll}
0_{3 \times 6} & I_{3 \times 3}
\end{array}\right] .
\end{gathered}
$$

The control scheme for augmented system (11) is thus designed as

$$
u\left(t_{k}\right)=\bar{K} \xi\left(t_{k}\right)=\left[\begin{array}{ll}
K & K_{e}
\end{array}\right]\left[\begin{array}{l}
x\left(t_{k}\right) \\
e\left(t_{k}\right)
\end{array}\right],
$$

where $K$ is defined as in (2) and $K_{e} \in \mathbb{R}^{m \times n}$ is to be designed. Substituting (13) into (11) yields the following augmented closed-loop system:

$$
\begin{gathered}
\dot{\xi}(t)=\bar{A} \xi(t)+\bar{B} \bar{K} \xi\left(t_{k}\right)+\bar{B}_{1} \bar{d}(t), \\
y(t)=\bar{C}_{1} \xi(t), \\
z(t)=\bar{C}_{2} \xi(t) .
\end{gathered}
$$

If system (14) is exponentially stable, then it implies that the relative position error integral action $e(t) \rightarrow 0$ and further implies that $\lim _{t \rightarrow \infty}=y_{r}$.

In the following discussion, we adopt the delay-input approach to deal with the sampled-data control problem. By defining

$$
\tau(t)=t_{k+1}-t_{k}
$$

the sampling intervals can be written as

$$
t_{k}=t-\left(t_{k+1}-t_{k}\right)=t-\tau(t) .
$$

Therefore, the sampled augmented state can be written as

$$
\xi\left(t_{k}\right)=\xi(t-\tau(t)),
$$

and the control input vector can be transformed into

$$
u\left(t_{k}\right)=\bar{K} \xi\left(t_{k}\right)=\bar{K} \xi(t-\tau(t)) .
$$

Hence, the augmented closed-loop system (14) can be written by

$$
\begin{gathered}
\dot{\xi}(t)=\bar{A} \xi(t)+\bar{B} \bar{K} \xi(t-\tau(t))+\bar{B}_{1} \bar{d}(t), \\
y(t)=\bar{C}_{1} \xi(t), \\
z(t)=\bar{C}_{2} \xi(t) .
\end{gathered}
$$

Now, the aim of this paper can be formulated as follows.

Problem 1. Design a sampled-data control law $u\left(t_{k}\right)$, such that the following conditions are met. 
(1) The closed-loop system (19) is exponentially stable with a given decay rate $\alpha>0$. In other words, the chaser holds at a relative position.

(2) The control input satisfies the given upper bound (8).

(3) For closed-loop system (19), the following $H_{\infty}$ performance index is achieved:

$$
e_{z}(t)=\|z(t)\|_{2}-\gamma\|\bar{d}(t)\|_{2} \leqslant 0
$$

\section{Main Results}

In this section, we will first perform the stability analysis and control scheme design for system (19) with $\bar{d}(t)=0$. Then, we will consider the internal stability of (19) with the prescribed $H_{\infty}$ performance index (20).

\subsection{Stabilization Analysis}

Theorem 2. Consider the closed-loop system (19) with $\bar{d}(t)=$ 0 , given a decay rate $\alpha>0$, if there exist positive definite matrices $P_{1}, P_{2}$, and $P_{3} \in \mathbb{R}^{9 \times 9}$, and matrices $T_{1}, T_{2}, S_{1}, S_{2}$, and $S_{3} \in \mathbb{R}^{9 \times 9}$, such that the following conditions hold:

$$
\begin{gathered}
\Phi=\left[\begin{array}{ccc}
\Phi_{11} & \Phi_{12} & \Phi_{13} \\
\star & \Phi_{22} & \Phi_{23} \\
\star & \star & \Phi_{33}
\end{array}\right]<0, \\
\widehat{\Phi}=\left[\begin{array}{cccc}
\widehat{\Phi}_{11} & \widehat{\Phi}_{12} & \widehat{\Phi}_{13} & \widehat{\Phi}_{14} \\
\star & \widehat{\Phi}_{22} & \widehat{\Phi}_{23} & 0 \\
\star & \star & \widehat{\Phi}_{33} & \widehat{\Phi}_{34} \\
\star & \star & \star & \widehat{\Phi}_{44}
\end{array}\right]<0, \\
\rho \bar{K}^{T} R_{i}^{T} R_{i} \bar{K}-u_{i, \text { max }}^{2} P_{1}<0,
\end{gathered}
$$

where $\rho$ is defined as in (47) and

$$
\begin{gathered}
\Phi_{11}=2 \alpha P_{1}-P_{3}+2 \alpha h P_{3}-T_{1}^{T} \bar{A}-\bar{A}^{T} T_{1}-S_{1}-S_{1}^{T}, \\
\Phi_{12}=P_{1}+T_{1}^{T}-h P_{3}, \\
\Phi_{13}=P_{3}-2 \alpha h P_{3}-T_{1}^{T} \bar{B} \bar{K}+S_{1}^{T}, \\
\Phi_{22}=h P_{2}+T_{2}+T_{2}^{T}, \\
\Phi_{23}=-h P_{3}-T_{2}^{T} \bar{B} \bar{K}+S_{2}+S_{2}^{T}, \\
\Phi_{33}=-P_{3}+2 \alpha h P_{3}+S_{3}+S_{3}^{T}, \\
\widehat{\Phi}_{11}=2 \alpha P_{1}-P_{3}-T_{1}^{T} \bar{A}-\bar{A} T_{1}-S_{1}-S_{1}^{T}, \\
\widehat{\Phi}_{12}=P_{1}+T_{1}^{T} \\
\widehat{\Phi}_{13}=P_{3}-T_{1}^{T} \bar{B} \bar{K}+S_{1}^{T}, \\
\widehat{\Phi}_{14}=h S_{1}^{T}, \\
\widehat{\Phi}_{22}=T_{2}^{T}+T_{2}^{T},
\end{gathered}
$$

$$
\begin{gathered}
\widehat{\Phi}_{23}=-T_{2}^{T} \bar{B} \bar{K}+S_{2}+S_{2}^{T}, \\
\widehat{\Phi}_{33}=-P_{3}+S_{3}+S_{3}^{T}, \\
\widehat{\Phi}_{34}=h S_{3}^{T}, \\
\widehat{\Phi}_{44}=-h e^{-2 \alpha h} P_{2} .
\end{gathered}
$$

Then system (19) with $\bar{d}(t)=0$ is exponentially stable with the decay rate $\alpha>0$.

Proof. For system (19), consider the following Lyapunov functional

$$
V(t)=V_{1}(t)+V_{2}(t)+V_{3}(t)
$$

where

$$
\begin{gathered}
V_{1}(t)=\xi^{T}(t) P_{1} \xi(t), \\
V_{2}(t)=(h-\tau(t)) \int_{t_{k}}^{t} \dot{\xi}^{T}(s) P_{2} \dot{\xi}(s) e^{-2 \alpha(t-s)} d s, \\
V_{3}(t)=(h-\tau(t))\left(\xi\left(t_{k}\right)-\xi(t)\right)^{T} P_{3}\left(\xi\left(t_{k}\right)-\xi(t)\right) .
\end{gathered}
$$

In function (3), $P_{i}>0(i=1,2,3)$ are Lyapunov matrices to be designed, and $\alpha>0$ is the decay rate as previously defined. It can be calculated that

$$
\begin{aligned}
\dot{V}_{1}(t)+ & 2 \alpha V_{1}(t)=2 \dot{\xi}^{T}(t) P_{1} \xi(t)+2 \alpha \xi^{T}(t) P_{1} \xi(t), \\
\dot{V}_{2}(t)= & -e^{-2 \alpha t} \int_{t_{k}}^{t} \dot{\xi}^{T}(s) P_{2} \dot{\xi}(s) e^{2 \alpha s} d s \\
& +(h-\tau(t)) e^{-2 \alpha t}(-2 \alpha) \\
& \times \int_{t_{k}}^{t} \dot{\xi}^{T}(s) P_{2} \dot{\xi}(s) e^{2 \alpha s} d s \\
= & (h-\tau(t)) e^{-2 \alpha t} \\
& \times\left(\dot{\xi}^{T}(t) P_{2} \dot{\xi}(t) e^{2 \alpha t}-\dot{\xi}^{T}\left(t_{k}\right) P_{2} \dot{\xi}\left(t_{k}\right) e^{2 \alpha t_{k}}\right)
\end{aligned}
$$

Notice that $\dot{\xi}^{T}\left(t_{k}\right) P_{2} \dot{\xi}\left(t_{k}\right) e^{2 \alpha t_{k}}=0$; thus,

$$
\begin{aligned}
\dot{V}_{2}(t)= & -e^{-2 \alpha t} \int_{t_{k}}^{t} \dot{\xi}^{T}(s) P_{2} \dot{\xi}(s) e^{2 \alpha s} d s \\
& -2 \alpha(h-\tau(t)) e^{-2 \alpha t} \int_{t_{k}}^{t} \dot{\xi}^{T}(s) P_{2} \dot{\xi}(s) e^{2 \alpha s} d s \\
& +(h-\tau(t)) \dot{\xi}^{T}(t) P_{2} \dot{\xi}(t) .
\end{aligned}
$$


Therefore, it is derived that

$$
\begin{aligned}
\dot{V}_{2}(t)+2 \alpha V_{2}(t)= & -e^{-2 \alpha t} \int_{t_{k}}^{t} \dot{\xi}^{T}(s) P_{2} \dot{\xi}(s) e^{2 \alpha s} d s \\
& -2 \alpha(h-\tau(t)) e^{-2 \alpha t} \\
& \times \int_{t_{k}}^{t} \dot{\xi}^{T}(s) P_{2} \dot{\xi}(s) e^{2 \alpha s} d s \\
& +(h-\tau(t)) \dot{\xi}^{T}(t) P_{2} \dot{\xi}(t) \\
& +2 \alpha(h-\tau(t)) e^{-2 \alpha t} \\
& \times \int_{t_{k}}^{t} \dot{\xi}^{T}(s) P_{2} \dot{\xi}(s) e^{2 \alpha s} d s \\
= & -e^{-2 \alpha t} \int_{t_{k}}^{t} \dot{\xi}^{T}(s) P_{2} \dot{\xi}(s) e^{2 \alpha s} d s \\
& +(h-\tau(t)) \dot{\xi}^{T}(t) P_{2} \dot{\xi}(t) .
\end{aligned}
$$

Furthermore, for $V_{3}(t)$ it is calculated directly that

$$
\begin{aligned}
\dot{V}_{3}(t)= & -\left(\xi\left(t_{k}\right)-\xi(t)\right)^{T} P_{3}\left(\xi\left(t_{k}\right)-\xi(t)\right) \\
& -(h-\tau(t)) \dot{\xi}^{T}(t) P_{3}\left(\xi\left(t_{k}\right)-\xi(t)\right) \\
& -(h-\tau(t))\left(\xi\left(t_{k}\right)-\xi(t)\right)^{T} P_{3} \dot{\xi}(t), \\
\dot{V}_{3}(t)+2 \alpha V_{3}(t)= & -\left(\xi\left(t_{k}\right)-\xi(t)\right)^{T} \\
& \times P_{3}\left(\xi\left(t_{k}\right)-\xi(t)\right) \\
& -(h-\tau(t)) \dot{\xi}^{T}(t) \\
& \times P_{3}\left(\xi\left(t_{k}\right)-\xi(t)\right) \\
& -(h-\tau(t)) \\
& \times\left(\xi\left(t_{k}\right)-\xi(t)\right)^{T} P_{3} \dot{\xi}(t) \\
& +2 \alpha(h-\tau(t))\left(\xi\left(t_{k}\right)-\xi(t)\right)^{T} \\
& \times P_{3}\left(\xi\left(t_{k}\right)-\xi(t)\right) .
\end{aligned}
$$

As a result, we have

$$
\begin{aligned}
\dot{V}(t)+2 \alpha V(t)= & \dot{V}_{1}(t)+2 \alpha V_{1}(t) \\
& +\dot{V}_{2}(t)+2 \alpha V_{2}(t) \\
& +\dot{V}_{3}(t)+2 \alpha V_{3}(t) \\
\leqslant & 2 \dot{\xi}^{T}(t) P_{1} \xi(t)+2 \alpha \xi^{T}(t) P_{1} \xi(t) \\
& -e^{-2 \alpha t} \int_{t_{k}}^{t} \dot{\xi}^{T}(s) P_{2} \dot{\xi}(s) e^{2 \alpha s} d s \\
& +(h-\tau(t)) \dot{\xi}^{T}(t) P_{2} \dot{\xi}(t)
\end{aligned}
$$

$$
\begin{aligned}
& -\left(\xi\left(t_{k}\right)-\xi(t)\right)^{T} \\
& \times P_{3}\left(\xi\left(t_{k}\right)-\xi(t)\right) \\
& -(h-\tau(t)) \dot{\xi}^{T}(t) \\
& \times P_{3}\left(\xi\left(t_{k}\right)-\xi(t)\right) \\
& -(h-\tau(t))\left(\xi\left(t_{k}\right)-\xi(t)\right)^{T} \\
& \times P_{3} \dot{\xi}(t)+2 \alpha(h-\tau(t)) \\
& \times\left(\xi\left(t_{k}\right)-\xi(t)\right)^{T} P_{3}\left(\xi\left(t_{k}\right)-\xi(t)\right) .
\end{aligned}
$$

We define the following variable

$$
\phi_{\xi}(t)=\frac{1}{\tau(t)} \int_{t_{k}}^{t} \dot{\xi}(s) d s,
$$

and it is derived that

$$
\begin{aligned}
& e^{-2 \alpha t} \int_{t_{k}}^{t} e^{2 \alpha s} \dot{\xi}^{T}(s) P_{2} \dot{\xi}(s) d s \\
& \geqslant e^{-2 \alpha t} e^{2 \alpha t_{k}} \int_{t_{k}}^{t} \dot{\xi}^{T}(s) P_{2} \dot{\xi}(s) \\
& =e^{-2 \alpha\left(t-t_{k}\right)} \int_{t_{k}}^{t} \dot{\xi}^{T}(s) P_{2} \dot{\xi}(s) \\
& =e^{-2 \alpha \tau(t)} \int_{t-\tau(t)}^{t} \dot{\xi}^{T}(s) P_{2} \dot{\xi}(s) .
\end{aligned}
$$

By Jensen's inequality, it is also shown that

$$
\begin{aligned}
\int_{t-\tau(t)}^{t} & \dot{\xi}^{T}(s) P_{2} \dot{\xi}(s) d s \\
\leqslant & \frac{1}{\tau(t)} \int_{t-\tau(t)}^{t} \dot{\xi}^{T}(s) d s \\
& \times P_{2} \int_{t-\tau(t)}^{t} \dot{\xi}(s) d s \\
= & \tau(t)\left(\frac{1}{\tau(t)} \int_{t-\tau(t)}^{t} \dot{\xi}^{T}(s) d s\right) \\
& \times P_{2}\left(\frac{1}{\tau(t)} \int_{t-\tau(t)}^{t} \dot{\xi}^{t}(s) d s\right) \\
= & \tau(t) \phi_{\xi}^{T}(t) P_{2} \phi_{\xi}(t) .
\end{aligned}
$$

In particular, for $\tau(t)=0$, it can be verified that the following holds:

$$
\begin{aligned}
& \left.\frac{1}{\tau(t)} \int_{t-\tau(t)}^{t} \dot{\xi}(s) d s\right|_{\tau(t)=0} \\
& \quad=\lim _{\tau(t) \rightarrow 0} \frac{1}{\tau(t)} \int_{t-\tau(t)}^{t} \dot{\xi}(s) d s=\dot{\xi}(t) .
\end{aligned}
$$


Therefore, it is shown from (30) that

$$
\begin{aligned}
\dot{V}(t)+2 \alpha V(t) \leqslant & 2 \dot{\xi}^{T}(t) P_{1} \xi(t) \\
& +2 \alpha \xi^{T}(t) P_{1} \xi(t) \\
& -e^{-2 \alpha \tau(t)} \tau(t) \phi_{\xi}^{T}(t) P_{2} \phi_{\xi}(t) \\
& +(h-\tau(t)) \dot{\xi}^{T}(t) P_{2} \dot{\xi}(t) \\
& -\left(\xi\left(t_{k}\right)-\xi(t)\right)^{T} P_{3}\left(\xi\left(t_{k}\right)-\xi(t)\right) \\
& -(h-\tau(t)) \dot{\xi}^{T}(t) P_{3}\left(\xi\left(t_{k}\right)-\xi(t)\right) \\
& -(h-\tau(t))\left(\xi\left(t_{k}\right)-\xi(t)\right)^{T} P_{3} \dot{\xi}(t) \\
& +2 \alpha(h-\tau(t))\left(\xi\left(t_{k}\right)-\xi(t)\right)^{T} \\
& \times P_{3}\left(\xi\left(t_{k}\right)-\xi(t)\right) .
\end{aligned}
$$

We now insert free-weighting matrices by introducing the following zero terms:

$$
\begin{aligned}
& 2\left[\xi^{T}(t) T_{1}^{T}+\dot{\xi}^{T}(t) T_{2}^{T}\right] \\
& \quad \times\left[\dot{\xi}^{T}(t)-\bar{A} \xi(t)-\bar{B} \bar{K} \xi\left(t_{k}\right)\right]=0, \\
& 2\left[\xi^{T}(t) S_{1}^{T}+\dot{\xi}^{T}(t) S_{2}^{T}+\xi^{T}\left(t_{k}\right) S_{3}^{T}\right] \\
& \quad \times\left[-\xi(t)+\xi\left(t_{k}\right)+\tau(t) \phi_{\xi}(t)\right]=0,
\end{aligned}
$$

where the free-weighting matrices $T_{1}, T_{2}, S_{1}, S_{2}$, and $S_{3}$ are to be designed. Then we have

$$
\begin{aligned}
\dot{V}(t) & +2 \alpha V(t) \\
\leqslant & 2 \dot{\xi}^{T}(t) P_{1} \xi(t)+2 \alpha \xi^{T}(t) P_{1} \xi(t) \\
& -e^{-2 \alpha \tau(t)} \tau(t) \phi_{\xi}^{T}(t) P_{2} \phi_{\xi}(t) \\
& +(h-\tau(t)) \dot{\xi}^{T}(t) P_{2} \dot{\xi}(t) \\
& -\left(\xi\left(t_{k}\right)-\xi(t)\right)^{T} P_{3}\left(\xi\left(t_{k}\right)-\xi(t)\right) \\
& -(h-\tau(t)) \dot{\xi}^{T}(t) P_{3}\left(\xi\left(t_{k}\right)-\xi(t)\right) \\
& -(h-\tau(t))\left(\xi\left(t_{k}\right)-\xi(t)\right)^{T} P_{3} \dot{\xi}(t) \\
& +2 \alpha(h-\tau(t))\left(\xi\left(t_{k}\right)-\xi(t)\right)^{T} \\
& \times P_{3}\left(\xi\left(t_{k}\right)-\xi(t)\right) \\
& +2\left[\xi^{T}(t) T_{1}^{T}+\dot{\xi}^{T}(t) T_{2}^{T}\right] \\
& \times\left[\dot{\xi}^{T}(t)-\bar{A} \xi(t)-\bar{B} \bar{K} \xi\left(t_{k}\right)\right] \\
& \times 2\left[\xi^{T}(t) S_{1}^{T}+\dot{\xi}^{T}(t) S_{2}^{T}+\xi^{T}\left(t_{k}\right) S_{3}^{T}\right] \\
& \times\left[-\xi(t)+\xi\left(t_{k}\right)+\tau(t) \phi_{\xi}(t)\right] .
\end{aligned}
$$

Defining the following new augmented variables

$$
\begin{aligned}
& \bar{\xi}(t)=\left[\begin{array}{llll}
\xi^{T}(t) & \dot{\xi}^{T}(t) & \xi^{T}\left(t_{k}\right) & \phi^{T}(t)
\end{array}\right]^{T}, \\
& \widehat{\xi}(t)=\left[\begin{array}{lll}
\xi^{T}(t) & \dot{\xi}^{T}(t) & \xi^{T}\left(t_{k}\right)
\end{array}\right]^{T},
\end{aligned}
$$

it is calculated that

$$
\dot{V}(t)+2 \alpha V(t) \leqslant \bar{\xi}^{T}(t) \Pi(t) \bar{\xi}(t)
$$

with

$$
\begin{gathered}
\Pi(t)=\left[\begin{array}{cccc}
\Pi_{11}(t) & \Pi_{12}(t) & \Pi_{13}(t) & \Pi_{14}(t) \\
\star & \Pi_{22}(t) & \Pi_{23}(t) & 0 \\
\star & \star & \Pi_{33}(t) & \Pi_{34}(t) \\
\star & \star & \star & \Pi_{44}(t)
\end{array}\right], \\
\Pi_{11}(t)=2 \alpha P_{1}-P_{3}+2 \alpha(h-\tau(t)) P_{3} \\
-T_{1}^{T} \bar{A}-\bar{A}^{T} T_{1}-S_{1}-S_{1}^{T}, \\
\Pi_{12}(t)=P_{1}+T_{1}^{T}-(h-\tau(t)) P_{3}, \\
\Pi_{13}(t)=P_{3}-2 \alpha(h-\tau(t)) P_{3}-T_{1}^{T} \bar{B} \bar{K}+S_{1}^{T}, \\
\Pi_{14}(t)=\tau(t) S_{1}^{T}, \\
\Pi_{22}(t)=(h-\tau(t)) P_{2}+T_{2}+T_{2}^{T}, \\
\Pi_{23}(t)=-(h-\tau(t)) P_{3}-T_{2}^{T} \bar{B} \bar{K}+S_{2}+S_{2}^{T}, \\
\Pi_{33}(t)=-P_{3}+2 \alpha(h-\tau(t)) P_{3}+S_{3}+S_{3}^{T}, \\
\Pi_{34}(t)=\tau(t) S_{3}^{T}, \\
\Pi_{44}(t)=-e^{-2 \alpha \tau(t)} \tau(t) P_{2} .
\end{gathered}
$$

We now consider the matrices $\Phi$ and $\widehat{\Phi}$ defined in (21). In fact, it can be verified that $\Phi$ is obtained from $\left.\Pi(t)\right|_{\tau(t)=0}$ with deleting the last row and last column, and $\widehat{\Phi}=\left.\Pi(t)\right|_{\tau(t)=h}$. Similar to the proof of Theorem 1 of [37], it can be proved that the system (19) with $\bar{d}(t)=0$ is exponentially stable.

Next, we deal with the input constraint along each axis. We define

$$
\begin{aligned}
& R_{x}=\left[\begin{array}{l}
1 \\
0 \\
0
\end{array}\right]\left[\begin{array}{lll}
1 & 0 & 0
\end{array}\right]=\left[\begin{array}{lll}
1 & 0 & 0 \\
0 & 0 & 0 \\
0 & 0 & 0
\end{array}\right], \\
& R_{y}=\left[\begin{array}{l}
0 \\
1 \\
0
\end{array}\right]\left[\begin{array}{lll}
0 & 1 & 0
\end{array}\right]=\left[\begin{array}{lll}
0 & 0 & 0 \\
0 & 1 & 0 \\
0 & 0 & 0
\end{array}\right], \\
& R_{z}=\left[\begin{array}{l}
0 \\
0 \\
1
\end{array}\right]\left[\begin{array}{lll}
0 & 0 & 1
\end{array}\right]=\left[\begin{array}{lll}
0 & 0 & 0 \\
0 & 0 & 0 \\
0 & 0 & 1
\end{array}\right] .
\end{aligned}
$$

Then, the thrust along each axis can be described as

$$
\left|u_{i}\left(t_{k}\right)\right|=\left\|R_{i} u\left(t_{k}\right)\right\| \leqslant u_{i, \max }, \quad i=x, y, z .
$$


Therefore, the thrust constraint (8) can be rewritten as

$$
\left\|R_{i} u\left(t_{k}\right)\right\| \leqslant u_{i, \max }, \quad i=x, y, z,
$$

which is equivalent to

$$
\left(R_{i} u\left(t_{k}\right)\right)^{T} R_{i} u\left(t_{k}\right) \leqslant u_{i, \max }^{2} .
$$

Furthermore, formula (45) is equal to

$$
\xi^{T}\left(t_{k}\right) \bar{K} R_{i}^{T} R_{i} \bar{K} \xi\left(t_{k}\right) \leqslant u_{i, \max }^{2}
$$

Based on the previous analysis, it is known that $\dot{V}(t)<0$ is guaranteed if the matrix conditions (21) hold. Subsequently, $V(t)<V(0)$ holds for any $t>0$. It is therefore reasonable to assume that there exists a scalar $\rho>0$ such that

$$
V(0) \leqslant \rho .
$$

Notice that $V(t)=V_{1}(t)+V_{2}(t)+V_{3}(t) \leq V(0) \leqslant \rho$, and $V_{2}(t) \geq 0, V_{3}(t) \geq 0$. Therefore, it is true that

$$
V(t) \leq V_{1}(t)<V(0) \leqslant \rho,
$$

which implies that

$$
V_{1}(t)<\rho
$$

Therefore, $\xi^{T}(t) P_{1} \xi(t)<\rho$ holds for any $t>0$. In particular, let $t=t_{k}$; we have

$$
\xi^{T}\left(t_{k}\right) P_{1} \xi\left(t_{k}\right)<\rho .
$$

Then, (46) can be guaranteed if (22) holds. We prove this fact. In fact, if (22) holds, we have

$$
\xi^{T}\left(t_{k}\right) \rho \bar{K}^{T} R_{i}^{T} R_{i} \bar{K} \xi\left(t_{k}\right)<\xi^{T}\left(t_{k}\right) u_{i, \max }^{2} P_{1} \xi\left(t_{k}\right) .
$$

By (51), we have

$$
\begin{aligned}
& \xi^{T}\left(t_{k}\right) \rho \bar{K}^{T} R_{i}^{T} R_{i} \bar{K} \xi\left(t_{k}\right) \\
& \quad<\xi^{T}\left(t_{k}\right) u_{i, \max }^{2} P_{1} \xi\left(t_{k}\right)<u_{i, \max }^{2} \rho,
\end{aligned}
$$

which implies that

$$
\xi^{T}\left(t_{k}\right) \bar{K}^{T} R_{i}^{T} R_{i} \bar{K} \xi\left(t_{k}\right)<u_{i, \max }^{2}
$$

As a result, the thrust limitation (8) can be guaranteed by (22). This completes the proof.

3.2. Disturbance Attenuation. In this subsection, we will investigate analysis of the internal stability and $H_{\infty}$ performance index for the system (19). Defining a new augmented vector $\psi(t) \triangleq\left[\xi^{T}(t) \bar{d}^{T}(t)\right]^{T}$, we reconsider the system (19) with $\bar{d}(t) \neq 0$. We introduce the performance index

$$
J(t)=\int_{0}^{t}\left[z^{T}(s) z(s)-\gamma \bar{d}^{T}(s) \bar{d}(s)\right] d s,
$$

Then, (20) can be guaranteed by $J(t)<0$.

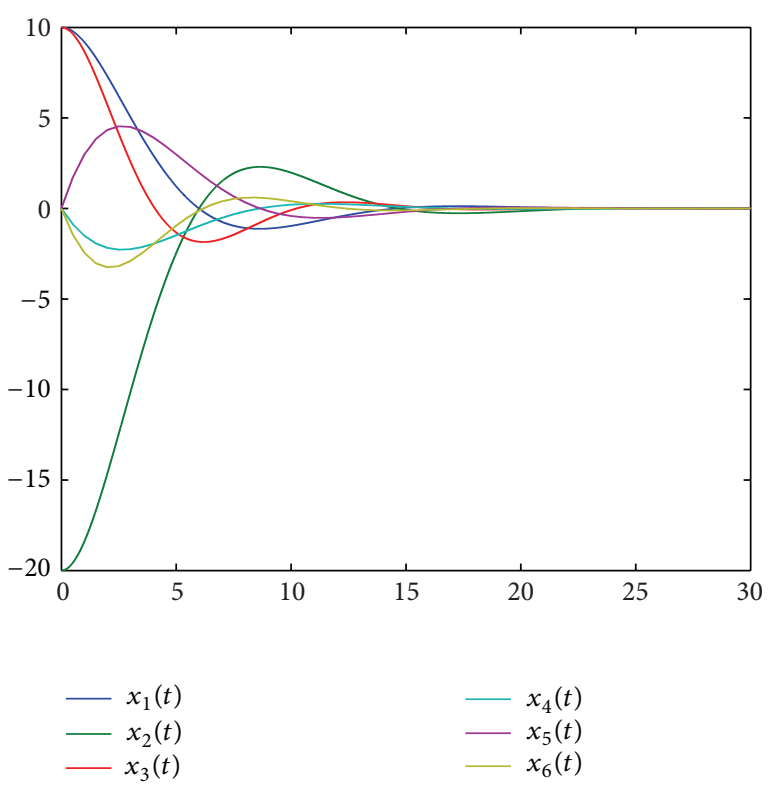

FIgURE 1: Trajectories of $x(t)$.

Theorem 3. Consider the closed-loop system (19), given a performance index parameter $\gamma>0$, let the decay rate $\alpha=0$, if there exist positive definite matrices $P_{1}, P_{2}$, and $P_{3} \in \mathbb{R}^{9 \times 9}$, and matrices $T_{1}, T_{2}, S_{1}, S_{2}$, and $S_{3} \in \mathbb{R}^{9 \times 9}$, such that the matrix conditions (21), (22) and the following matrices conditions hold:

$$
\begin{aligned}
& {\left[\begin{array}{cc}
\left.\Phi\right|_{\alpha=0} & \Gamma_{1} \\
\star & \operatorname{diag}\left\{-\gamma^{2},-I_{9}\right\}
\end{array}\right]<0,} \\
& {\left[\begin{array}{cc}
\left.\widehat{\Phi}\right|_{\alpha=0} & \Gamma_{2} \\
\star & \operatorname{diag}\left\{-\gamma^{2},-I_{9}\right\}
\end{array}\right]<0,}
\end{aligned}
$$

$$
\Gamma_{1}=\left[\begin{array}{cc}
-T_{1}^{T} \bar{B}_{1}^{T} & C_{2}^{T} \\
-T_{2}^{T} \bar{B}_{1}^{T} & 0 \\
0 & 0
\end{array}\right] \text {, }
$$

$$
\Gamma_{2}=\left[\begin{array}{cc}
-T_{1}^{T} \bar{B}_{1}^{T} & C_{2}^{T} \\
-T_{2}^{T} \bar{B}_{1}^{T} & 0 \\
0 & 0 \\
0 & 0
\end{array}\right] \text {. }
$$

Then the system (19) is internally stable, and the $H_{\infty}$ performance index (20) is achieved.

Proof. It is well known that $\dot{V}(t)<0$ implies that $\lim _{t \rightarrow \infty} V(t)=0$. We consider a new defined function

$$
V_{J}(t)=\dot{V}(t)+z^{T}(t) z(t)-\gamma^{2} \bar{d}^{T}(t) \bar{d}(t) .
$$

If $V_{J}(t)<0$, it is shown that

$$
z^{T}(t) z(t)-\gamma^{2} \bar{d}^{T}(t) \bar{d}(t)<-\dot{V}(t),
$$




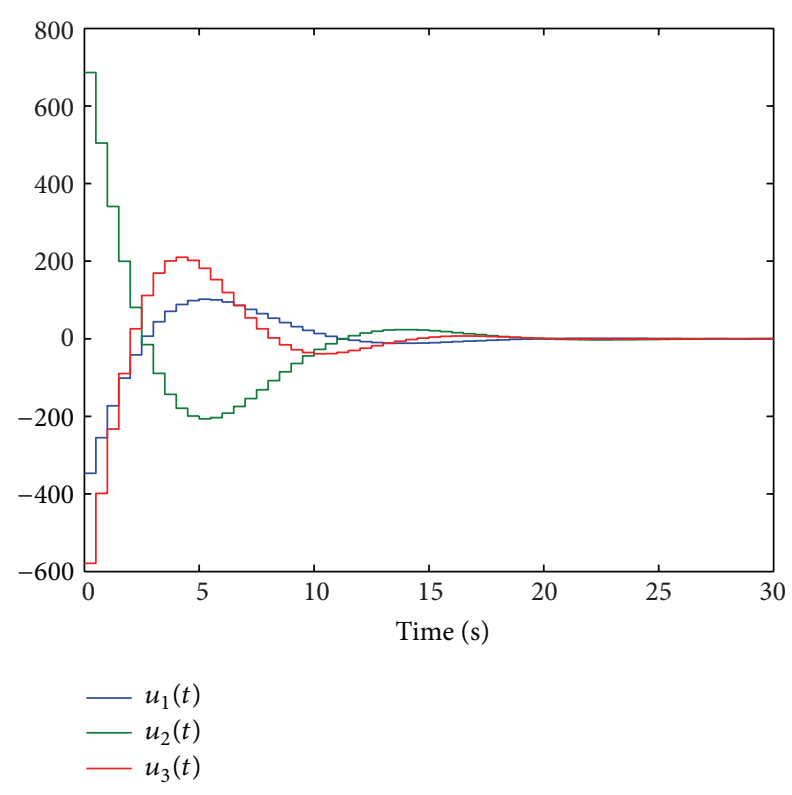

FIGURE 2: Trajectories of $u(t)$.

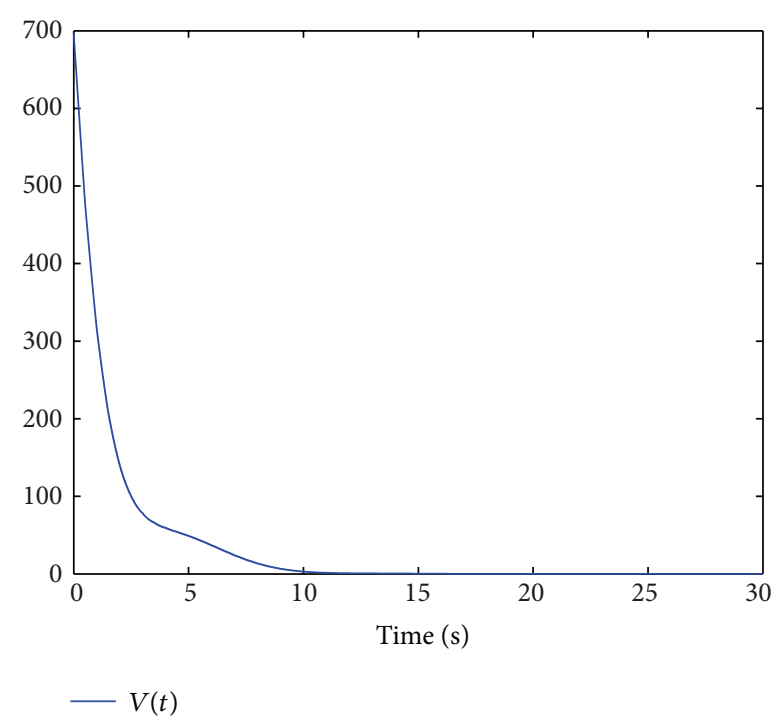

FIGURE 3: Lyapunov function $V(t)$.

$$
\bar{K}=\left[\begin{array}{ccccccccc}
-34.3807 & 0.1490 & 0 & -97.3889 & 0.0051 & 0 & -8.7062 & 0.0254 & 0 \\
-0.1490 & -34.3801 & 0 & -0.0051 & -97.3880 & 0 & -0.0254 & -8.7062 & 0 \\
0 & 0 & -57.8745 & 0 & 0 & -110.0009 & 0 & 0 & -16.5843
\end{array}\right]
$$

and it further implies that $J(\infty)=\int_{0}^{\infty}\left(z^{T}(s) z(s)-\gamma^{2}\right.$ $\left.\bar{d}^{T}(s) \bar{d}(s)\right) d s<-\int_{0}^{\infty} \dot{V}(s) d s=V(\infty)-V(0)=-V(0)<0$. That means, in the case $\alpha=0$, if $\dot{V}(t)<0$ and $V_{J}(t)<0$ hold, it can be guaranteed that the system (19) is internally stable and the $H_{\infty}$ performance index (20) can be achieved successfully.

Notice that (57) can be rewritten as

$$
\dot{V}(t)+\xi^{T}(t) \bar{C}_{2}^{T} \bar{C}_{2} \xi(t)-\gamma^{2} \bar{d}^{T}(t) \bar{d}(t)<0 .
$$

Recall the zero terms (37) and notice that the term (37) with $\bar{d}(t) \neq 0$ becomes

$$
\begin{aligned}
& 2\left[\xi^{T}(t) T_{1}^{T}+\dot{\xi}^{T}(t) T_{2}^{T}\right] \\
& \quad \times\left[\dot{\xi}^{T}(t)-\bar{A} \xi(t)-\bar{B} \bar{K} \xi\left(t_{k}\right)-\bar{B}_{1} \bar{d}(t)\right]=0, \\
& 2\left[\xi^{T}(t) S_{1}^{T}+\dot{\xi}^{T}(t) S_{2}^{T}+\xi^{T}\left(t_{k}\right) S_{3}^{T}\right] \\
& \quad \times\left[-\xi(t)+\xi\left(t_{k}\right)+\tau(t) \phi_{\xi}(t)\right]=0 .
\end{aligned}
$$

By similar derivation of Theorem 2, it can be proved that system (19) is internally stable if the conditions (55) hold. On the other hand, it has been proved in Theorem 2 that $\dot{V}(t)<0$ is guaranteed by (21)-(22). This completes the proof.

\section{Simulation}

In this section, simulation results will be shown to illustrate the usefulness and advantage of the proposed sampled-data control approach. We consider the numerical example in [3]: suppose that the mass of the chaser is $200 \mathrm{~kg}$, that is, $m=200$, and the target is moving along a geosynchronous orbit with radius $r=42241 \mathrm{~km}$ with an orbital period of $24 \mathrm{~h}$. The angle velocity $w=1.117 \times 10.3 \mathrm{rad} / \mathrm{s}$. The maximum thrust along each axis is $1000 \mathrm{~N}$. The disturbance vector is described as $d(t)=\left[\begin{array}{lll}2 \sin (2 t) & 2 \sin (2 t) & 2 \sin (2 t)\end{array}\right]^{T}$. The upper bound of the sampling period is given as $h=0.5$, and the decay rate is selected as $\alpha=0.02$. The disturbance attenuation level is given as $\gamma=0.4$. In the coordinate based on the target frame, assume that the initial state is $[10,-20,10,0,0,0]$, and the desired holding position is $(0,-1,0)$. Design the state feedback gain $\bar{K}$ as

The simulation results are provided in Figures 1-3, which show the effectiveness of the proposed methods. The trajectories of state variable $x(t)$ with $d(t)=0$ are shown in Figure 1; the trajectories of sampled-data control input $u(t)$ with $d(t)=0$ are shown in Figure 2; the trajectories of Lyapunov functions $V(t)$ with $d(t)=0$ are shown in Figure 3. It can be seen that the obtained performance is desirable. such that $(\bar{A}+\bar{B} \bar{K})$ is Hurwitz. Suppose that $t_{1}=0$, which means that the first sampling time instant is the initial time. The designed parameter $\rho$ is chosen as $\rho=950$. Solving the conditions (21)-(22) and (55), it is found that there exists feasible solution. Then, it can be derived that $V(0)=V_{1}(0)=$ $x(0)^{T} P_{1} x(0)=835.9423$. It is obvious the condition (47) holds, which means that our obtained solution is correct and feasible. 


\section{Conclusion}

In this paper, we have addressed the problem of sampleddata stabilization for spacecraft rendezvous with a timedependent Lyapunov approach. A discontinuous Lyapunov functional has been constructed, the value of which does not increase in each sampling time instant. The sufficient conditions for the stability analysis and controller design are established in terms of matrix inequality technique. Future work will be focused on more complicated case; that is, the effects of network-induced phenomenon such as communication delay and packet losses are taken into account simultaneously.

\section{Acknowledgments}

This work was partially supported by the National Natural Science Foundation of China 61104101, the Fundamental Research Funds for the Central Universities, the China Postdoctoral Science Foundation funded project (2011M500058), the Special Chinese National Postdoctoral Science Foundation under Grant 2012T50356, and the Heilongjiang Postdoctoral Fund (LBH-Z11144).

\section{References}

[1] U. Ahsun, D. W. Miller, and J. L. Ramirez, "Control of electromagnetic satellite formations in near-Earth orbits," Journal of Guidance, Control, and Dynamics, vol. 33, no. 6, pp. 1883-1891, 2010.

[2] H. Schaub, "Stabilization of satellite motion relative to a Coulomb spacecraft formation," Journal of Guidance, Control, and Dynamics, vol. 28, no. 6, pp. 1231-1239, 2005.

[3] X. Yang, X. Cao, and H. Gao, "Sampled-data control for relative position holding of spacecraft rendezvous with thrust nonlinearity," IEEE Transactions on Industrial Electronics, vol. 59, no. 2, pp. 1146-1153, 2011.

[4] P. Gurfil, "Nonlinear feedback control of low-thrust orbital transfer in a central gravitational field," Acta Astronautica, vol. 60, no. 8-9, pp. 631-648, 2007.

[5] S. Kurnaz, O. Cetin, and O. Kaynak, "Fuzzy logic based approach to design of flight control and navigation tasks for autonomous unmanned aerial vehicles," Journal of Intelligent and Robotic Systems, vol. 54, no. 1-3, pp. 229-244, 2009.

[6] W. Clohessy and R. Wiltshire, "Terminal guidance system for satellite rendezvous," Journal of the Aerospace Sciences, vol. 27, no. 9, pp. 653-658, 1960.

[7] Y. Luo and G. Tang, "Spacecraft optimal rendezvous controller design using simulated annealing," Aerospace Science and Technology, vol. 9, no. 8, pp. 732-737, 2005.

[8] Y. Luo, G. Tang, and Y. Lei, "Optimal multi-objective linearized impulsive rendezvous," Journal of Guidance, Control, and Dynamics, vol. 30, no. 2, pp. 383-389, 2007.

[9] Z. Mao and B. Jiang, "Fault identification and fault-tolerant control for a class of networked control systems," International Journal of Innovative Computing, Information and Control, vol. 3, no. 5, pp. 1121-1130, 2007.

[10] X. Su, P. Shi, L. Wu, and Y. Song, "A novel control design on discrete-time Takagi-Sugeno fuzzy systems with time-varying delays," IEEE Transactions on Fuzzy Systems, vol. 21, no. 4, pp. 655-671, 2012.
[11] X. Su, L. Wu, P. Shi, and Y. Song, " $H_{\infty}$ model reduction of T-S fuzzy stochastic systems," IEEE Transactions on Systems, Man, and Cybernetics B, vol. 42, no. 6, pp. 1574-1585, 2012.

[12] S. Yin, S. Ding, and H. Luo, "Real-time implementation of fault tolerant control system with performance optimization," IEEE Transactions on Industrial Electronics, 2013.

[13] R. Yang, Z. Zhang, and P. Shi, "Exponential stability on stochastic neural networks with discrete interval and distributed delays," IEEE Transactions on Neural Networks, vol. 21, no. 1, pp. 169-175, 2010.

[14] R. Yang, P. Shi, G.-P. Liu, and H. Gao, "Network-based feedback control for systems with mixed delays based on quantization and dropout compensation," Automatica, vol. 47, no. 12, pp. 2805-2809, 2011.

[15] R. Yang, P. Shi, and G.-P. Liu, "Filtering for discrete-time networked nonlinear systems with mixed random delays and packet dropouts," IEEE Transactions on Automatic Control, vol. 56, no. 11, pp. 2655-2660, 2011.

[16] Y. Shu, L. Yu, and Z. Wenan, "Estimator-based control of networked systems with packet-dropouts," International Journal of Innovative Computing, Information and Control, vol. 6, no. 6, pp. 2737-2748, 2010.

[17] C. Jiang, Q. Zhang, and D. Zou, "Delay-dependent robust filtering for networked control systems with polytopic uncertainties," International Journal of Innovative Computing, Information and Control, vol. 6, no. 11, pp. 4857-4868, 2010.

[18] S. Shi, Z. Yuan, and Q. Zhang, "Fault-tolerant $H_{\infty}$ filter design of a class of switched systems with sensor failures," International Journal of Innovative Computing, Information and Control, vol. 5, no. 11, pp. 3827-3838, 2009.

[19] S. Yin, S. Ding, A. Haghani, H. Hao, and P. Zhang, "A comparison study of basic data-driven fault diagnosis and process monitoring methods on the benchmark Tennessee Eastman process," Journal of Process Control, vol. 22, no. 9, pp. 1567-1581, 2012.

[20] J. Lu, D. W. C. Ho, and J. Cao, "A unified synchronization criterion for impulsive dynamical networks," Automatica, vol. 46, no. 7, pp. 1215-1221, 2010.

[21] J. Lu, D. W. C. Ho, J. Cao, and J. Kurths, "Exponential synchronization of linearly coupled neural networks with impulsive disturbances," IEEE Transactions on Neural Networks, vol. 22, no. 2, pp. 329-336, 2011.

[22] H. Wu and M. Bai, "Stochastic stability analysis and synthesis for nonlinear fault tolerant control systems based on the T-S fuzzy model," International Journal of Innovative Computing, Information and Control, vol. 6, no. 9, pp. 3989-4000, 2010.

[23] S. Yin, Data-Driven Design of Fault Diagnosis Systems, VDI, Duesseldorf, Germany, 2012.

[24] S. Yin, S. X. Ding, A. H. A. Sari, and H. Hao, "Data-driven monitoring for stochastic systems and its application on batch process," International Journal of Systems Science, vol. 44, no. 7, pp. 1366-1376, 2013.

[25] L. Wu and W. X. Zheng, "Passivity-based sliding mode control of uncertain singular time-delay systems," Automatica, vol. 45, no. 9, pp. 2120-2127, 2009.

[26] L. Wu, P. Shi, and H. Gao, "State estimation and slidingmode control of Markovian jump singular systems," IEEE Transactions on Automatic Control, vol. 55, no. 5, pp. 1213-1219, 2010.

[27] L. Zhang and E.-K. Boukas, "Stability and stabilization of Markovian jump linear systems with partly unknown transition probabilities," Automatica, vol. 45, no. 2, pp. 463-468, 2009. 
[28] L. Zhang and E.-K. Boukas, "Mode-dependent $H_{\infty}$ filtering for discrete-time Markovian jump linear systems with partly unknown transition probabilities," Automatica, vol. 45, no. 6, pp. 1462-1467, 2009.

[29] L. Zhou and G. Lu, "Quantized feedback stabilization for networked control systems with nonlinear perturbation," International Journal of Innovative Computing, Information and Control, vol. 6, no. 6, pp. 2485-2496, 2010.

[30] Z.-G. Wu, J. H. Park, H. Su, and J. Chu, "Discontinuous Lyapunov functional approach to synchronization of time-delay neural networks using sampled-data," Nonlinear Dynamics, vol. 69, no. 4, pp. 2021-2030, 2012.

[31] H. Li, H. Liu, H. Gao, and P. Shi, "Reliable fuzzy control for active suspension systems with actuator delay and fault," IEEE Transactions on Fuzzy Systems, vol. 20, no. 2, pp. 342-357, 2012.

[32] H. Li, J. Yu, C. Hilton, and H. Liu, "Adaptive sliding mode control for nonlinear active suspension systems using T-S fuzzy model," IEEE Transactions on Industrial Electronics, vol. 60, no. 8, pp. 3328-3338, 2012.

[33] E. Fridman, "A refined input delay approach to sampled-data control," Automatica, vol. 46, no. 2, pp. 421-427, 2010.

[34] E. Fridman, A. Seuret, and J.-P. Richard, "Robust sampleddata stabilization of linear systems: an input delay approach," Automatica, vol. 40, no. 8, pp. 1441-1446, 2004.

[35] P. Naghshtabrizi, J. P. Hespanha, and A. R. Teel, "Exponential stability of impulsive systems with application to uncertain sampled-data systems," Systems \& Control Letters, vol. 57, no. 5, pp. 378-385, 2008.

[36] M. Liu, J. You, and X. Ma, " $H_{\infty}$ filtering for sampled-data stochastic systems with limited capacity channel," Signal Processing, vol. 91, no. 8, pp. 1826-1837, 2011.

[37] M. Liu, D. W. C. Ho, and Y. Niu, "Observer-based controller design for linear systems with limited communication capacity via a descriptor augmentation method," IET Control Theory \& Applications, vol. 6, no. 3, pp. 437-447, 2012. 


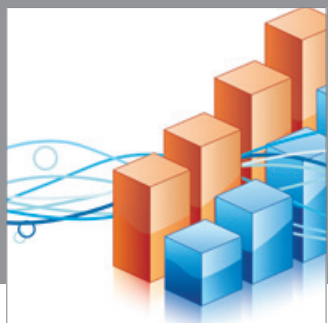

Advances in

Operations Research

mansans

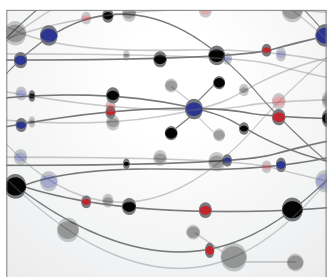

The Scientific World Journal
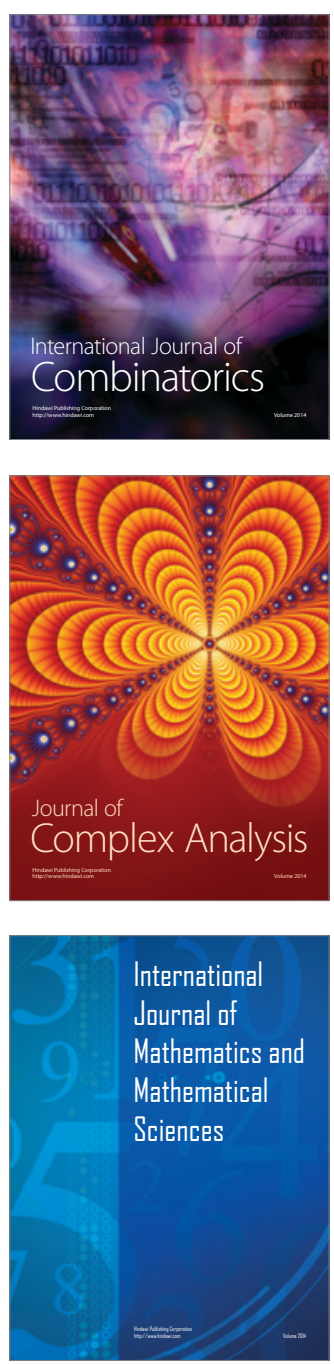
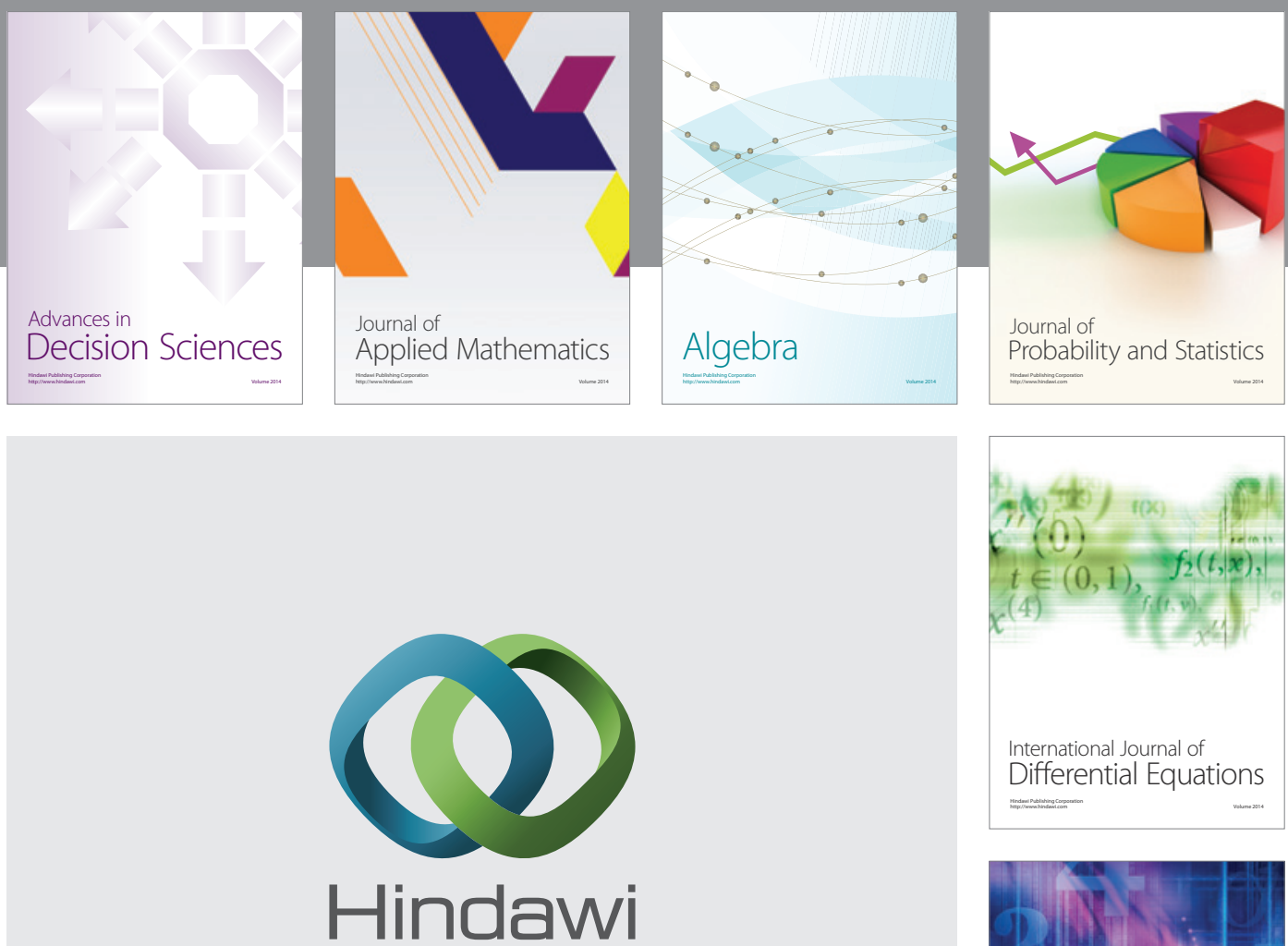

Submit your manuscripts at http://www.hindawi.com
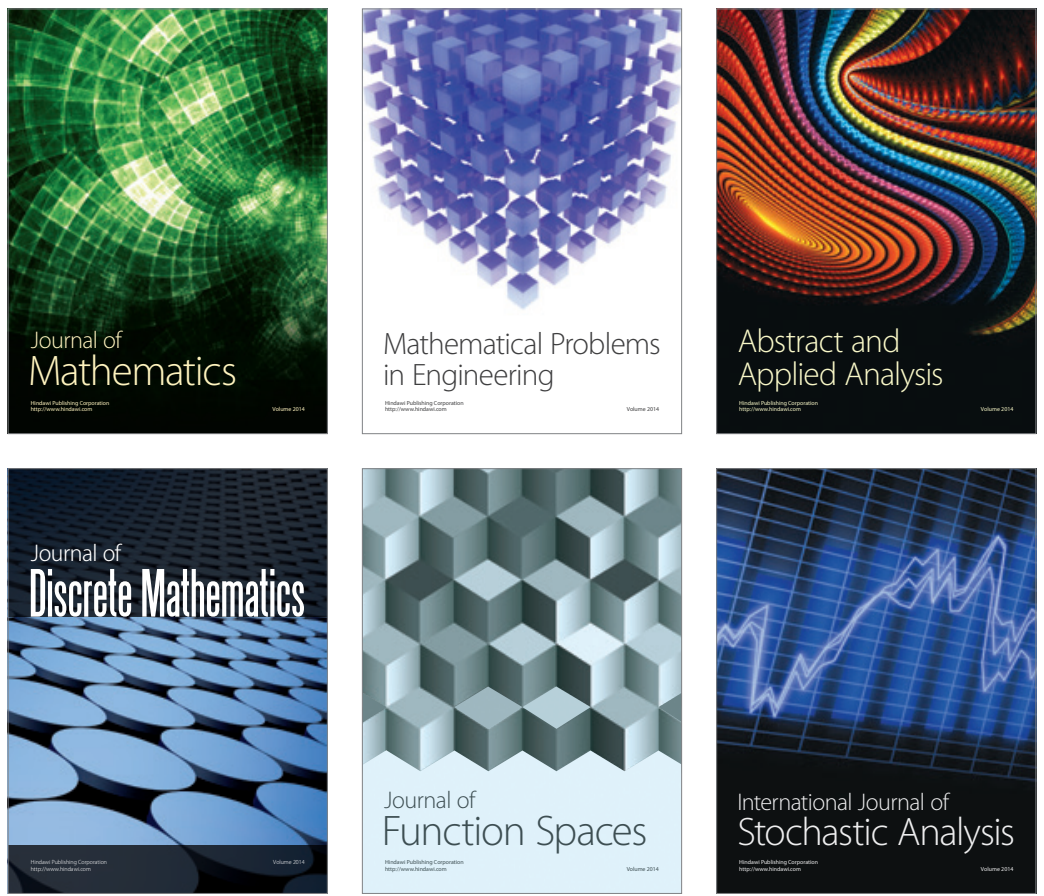

Journal of

Function Spaces

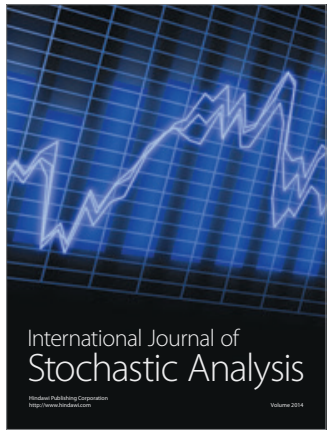

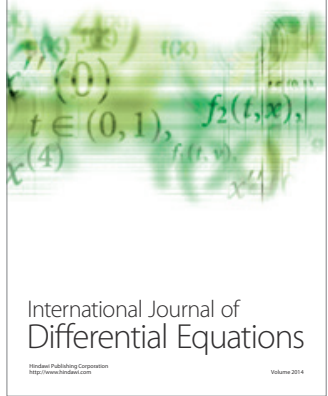
